Open Access

\title{
Healthcare decision-making in end stage renal disease-patient preferences and clinical correlates
}

Anuradha Jayanti ${ }^{* *}$, Markus Neuvonen ${ }^{2}$, Alison Wearden ${ }^{3}$, Julie Morris ${ }^{4}$, Philip Foden $^{4}$, Paul Brenchley ${ }^{1}$, Sandip Mitra ${ }^{1}$ and on behalf of the BASIC-HHD study group

\begin{abstract}
Background: Medical decision-making is critical to patient survival and well-being. Patients with end stage renal disease (ESRD) are faced with incrementally complex decision-making throughout their treatment journey. The extent to which patients seek involvement in the decision-making process and factors which influence these in ESRD need to be understood.

Methods: 535 ESRD patients were enrolled into the cross-sectional study arm and 30 patients who started dialysis were prospectively evaluated. Patients were enrolled into 3 groups- 'predialysis' (group A), 'in-centre' haemodialysis (HD) (group B) and self-care HD (93\% at home-group C) from across five tertiary UK renal centres. The Autonomy Preference Index (API) has been employed to study patient preferences for information-seeking (IS) and decision-making (DM). Demographic, psychosocial and neuropsychometric assessments are considered for analyses.
\end{abstract}

Results: 458 complete responses were available. API items have high internal consistency in the study population (Cronbach's alpha $>0.70$ ). Overall and across individual study groups, the scores for information-seeking and decision-making are significantly different indicating that although patients had a strong preference to be well informed, they were more neutral in their preference to participate in DM $(p<0.05)$. In the age, education and study group adjusted multiple linear regression analysis, lower age, female gender, marital status; higher API IS scores and white ethnicity background were significant predictors of preference for decision-making. DM scores were subdivided into tertiles to identify variables associated with high $(\mathrm{DM}>70$ : and low DM $(\leq 30)$ scores. This shows association of higher DM scores with lower age, lower comorbidity index score, higher executive brain function, belonging in the self-caring cohort and being unemployed. In the prospectively studied cohort of predialysis patients, there was no change in decision-making preference scores after commencement of dialysis.

Conclusion: ESRD patients prefer to receive information, but this does not always imply active involvement in decision-making. By understanding modifiable and non-modifiable factors which affect patient preferences for involvement in healthcare decision-making, health professionals may acknowledge the need to accommodate individual patient preferences to the extent determined by the individual patient factors.

Keywords: Haemodialysis, Autonomy, Decision-making, Cognition

\footnotetext{
* Correspondence: anu_5577@yahoo.co.in

${ }^{1}$ Department of Nephrology, Central Manchester Hospitals NHS Trust,

Manchester Royal Infirmary, Oxford Road, Manchester M13 9WL, UK

Full list of author information is available at the end of the article
}

(c) 2015 Jayanti et al. Open Access This article is distributed under the terms of the Creative Commons Attribution 4.0 International License (http://creativecommons.org/licenses/by/4.0/), which permits unrestricted use, distribution, and reproduction in any medium, provided you give appropriate credit to the original author(s) and the source, provide a link to the Creative Commons license, and indicate if changes were made. The Creative Commons Public Domain Dedication waiver (http://creativecommons.org/publicdomain/zero/1.0/) applies to the data made available in this article, unless otherwise stated. 


\section{Background}

'Nothing is more difficult, and therefore more precious, than to be able to decide'

Napoleon Bonaparte

Medical decision-making is critical to patient survival and well-being[1]. Over the last two decades, the convergence of influential ideas from the fields of bioethics, psychology, sociology and medicine has contributed to our understanding of the beneficial role of engaging patients in the medical decision-making process. The several potential benefits of involving patients in medical decision-making (DM) include reduced anxiety and depression, greater self-efficacy, improved concordance, and higher satisfaction with their physician [2-6]. Patient's expectations about exercising choice in medical decision-making have also been influenced by socio-cultural factors. These stem from increasing consumerist attitudes and litigious practices in the society, leading to the belief amongst healthcare professionals, that patients are best placed to evaluate the risks and benefits of alternative treatments [7], [8]. Of the models of healthcare decisionmaking that exist, an extreme and impractical version of the 'patient engagement model' of healthcare in practice would result in the providers supplying accurate information to patients without sharing their own views or experiences and then expecting patients to make tough medical decisions on their own. Research has demonstrated that patients' desire for information is typically underestimated by physicians[6]. What is less apparent is the extent to which they seek involvement in the actual decision-making process.

Healthcare decision-making is a highly complex process, the outcome of which is the interplay of several interrelated factors $[7,9,10]$ and not limited only to uncertainty in scientific evidence. As decisionmaking is affected by several factors, it is prone to error[1]. It is not surprising therefore, as to why some patient decisions may be at odds with the healthcare provider recommendations, making even shared decisions difficult to implement in clinical practice.

In several clinical conditions, evidence shows that not all patients want to make their own decisions[6] and some would actively delegate the task to their healthcare professionals. This concept has not been well understood in chronic kidney disease (CKD). Patients with CKD are faced with incrementally complex decisionmaking throughout their treatment journey. Particularly in later stages, patients exercise choice and make decisions which impact on how they live from day to day. Some of these include decisions around dietary intake, medications, frequency of clinic visits, treatment options when they reach end stage renal disease (ESRD) and even the choice of not considering renal replacement therapy. Dialysis, a life-sustaining therapy, invites multiple levels of patient engagement with and without healthcare providers, making it an intellectually and emotionally demanding process. Accommodating individual patient preferences for participation and true shared decision-making as the 'ideal' may be in potential conflict in some instances.

'Autonomy' in decision-making is one of several factors which may influence healthcare decisions throughout the ESRD journey. We chose to study this construct to understand its basis in undertaking self-care in the ESRD context. Decisions are taken based on the information patients acquire from healthcare providers and other sources. Also, the impact of 'real' vs 'imaginary' knowledge may influence patient attitudes to decisionmaking. Patients with ESRD are expected to assimilate a lot of new information in a particularly vulnerable phase of their illness, sometimes with limitations in cognitive and computational skills [11] and in relatively short time frames, leading to critical, life-changing decisions. Multiple inter-related skills are required to function optimally and produce the best outcomes for the individual circumstance. These include the ability to access and comprehend information, recall the same, weigh alternatives, infer and communicate decisions effectively and engage in a life-long process of learning [12, 13]. All of these activities are a product of complex processing of information in the brain of individuals. Executive brain function is a higher order cognitive ability that is a product of working memory, reasoning, task flexibility and visuo-motor speed. It is well known that chronic kidney disease is associated with considerable executive and episodic memory cognitive deficit, which is also progressively on the decline, after commencement of haemodialysis [11]. The reported prevalence of cognitive deficit in dialysis patients is of the order of $17-50 \%$ [14]. Closely related to systematic, careful cognitive processing is the role of the patient's 'affect' on decision-making. Understanding ESRD healthcare decision-making from a psychological perspective is paramount due to the high prevalence of depression or anxiety amongst these patients (up to $70 \%$ ) $[15,16]$.

\section{Study objectives}

Although 'autonomy' and 'decision-making' are not synonymous with each other, in contemporary medical literature, the two have been used interchangeably[24]. In the present study, 'information-seeking' and 'decisionmaking' preferences are evaluated in a large group of ESRD patients.

We sought to

1) Describe the properties of Autonomy Preference Index (API) instrument in ESRD population. 
2) Examine clinical, psychological and neurocognitive correlates of 'autonomous decision-makers' vs 'delegators' in ESRD.

3) Study the impact of commencement of dialysis on decision-making, in a subset of predialysis patients.

\section{Methods}

The API study data are derived from data ascertained for the BASIC-HHD study[18]. The BASIC-HHD study is a comprehensive and systematic study of barriers and enablers of the uptake and maintenance of home HD therapy. The study involves five UK centres, with variable prevalence rates of home HD. The centres reported similar structure of pre-dialysis education programmes with access to nurse specialists for information and dedicated 'low-clearance' clinics. An integrated mixed methodology (convergent, parallel design) has been adopted for the BASIC-HHD study in a combined cross-sectional and prospective study design. The methodological details and scope of data collected in the BASIC-HHD appear in a published protocol[18].

\section{Study registration}

This study has been reviewed and approved by the Greater Manchester West Health Research Authority National Research Ethics Service (NRES) Reference number: 12/ $\mathrm{NW} / 0170$. The study is on the NIHR portfolio (ID 12346). Written, informed consent from participants was obtained for the study

\section{Participants}

Data presented here are derived from the cross-sectional and prospective segments of the BASIC-HHD study. 535 patients were enrolled in three groups. Predialysis patients for the CKD-5 group (group A), prevalent incentre' HD patients (group B) were approached if they fulfilled eligibility criteria and were willing to undertake neuropsychometric assessments and complete study specific questionnaires. All self-care haemodialysis patients (93\% at home) from each participating centre were also approached (group C). Predialysis patients were approached consecutively from the predialysis clinics and hospital haemodialysis patients were approached in consecutive order across all shifts until the centre target for recruitment was reached. Most participants approached were willing to engage with the study and reasons for declining participation included a lack of interest in research participation, and 'research' fatigue.

\section{Procedure}

Psychological measures employed in this study were a part of compilation of questionnaires. Blood sampling and neuropsychometric assessments were carried out ahead of the dialysis sessions. HD patients returned the questionnaires on the same day or within a couple of dialysis sessions 'in-centre'. Home HD patients returned it by post, as did the pre-dialysis patients. Visually impaired patients could respond to questions posed to them by the research team member.

\section{Measures}

The Autonomy Preference Index was used to study patient preferences for information-seeking and decisionmaking. This tool was developed and validated originally in a group of general medical patients [17]. This tool consists of two subscales: an eight-item informationseeking subscale and a six-item decision-making subscale. The format of the responses is on a 5-point Likert scale. Scores for both domains are linearized to range from 0-100 (percentage scores), with higher scores indicating stronger preferences for participation. In addition, in the original API there are eight items corresponding to three clinical vignettes representing increasing disease severity to assess if symptom severity plays a role in patient autonomy preferences. The API has been validated and utilized in numerous other patient populations. The tool was employed; unmodified, as the questions and clinical scenarios are both relevant and not unfamiliar to the ESRD population.

Additionally, all study participants completed a compilation of questionnaires[18]. In order to examine the potential impact of patient's affect and cognitive ability on their engagement with decision-making, additional instruments analysed in the present study are the Beck Depression Inventory II[19] and the State and Trait Anxiety Inventory[20]. Participants underwent cognitive assessment using the modified mini-mental state examination (3MS)[21], and trail making tests A and B (TMTA/TMTB) scores[22]. The scores from these instruments were considered in ordered categories for analyses: BDI $(0-10,11-15,16-20,21-25$, 26-30, 31+), STAI $(20-29,30-39,40-49,50+)$ and $3 \mathrm{MS}$ (94-100, 86-93, 81-85, 76-80, $\leq 75)$.

\section{Missing data}

Overall the study had excellent data completion across all instruments used in the study ( $>82 \%)$. The API subscales were complete in $85.6 \%$ of the cases (Fig. 1). The only statistically significant difference between those who were missing both the API decision-making and API information-seeking scores $(n=77)$ and those who were not missing both is in ethnicity. Non-white patients were more likely not to complete both API scores than white patients. Ethnicity was associated with decision making in the final multivariable analysis for the decision making variable. Therefore, there is a chance that the point estimate may change slightly, depending on whether the non-white patients who responded had different scores to those who did not respond. However, 


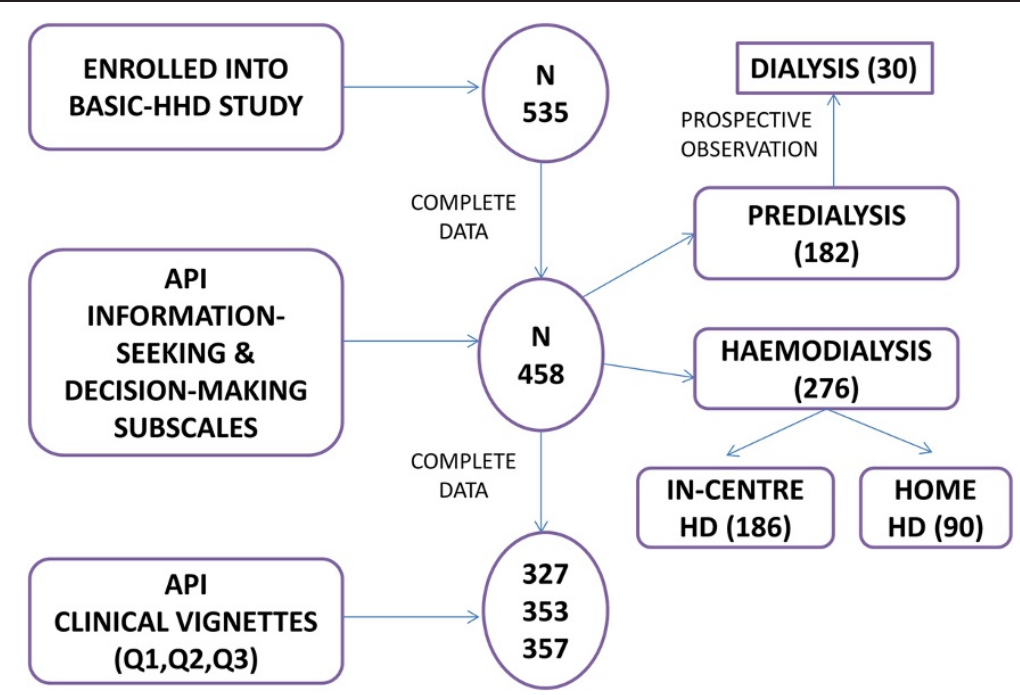

Fig. 1 Diagram depicting API data available for analysis (N)

with the relatively small amount of missing data and only 15 non-white patients not having either score, any change would be small. There was no relationship in the single variable analysis between ethnicity and information seeking so unless the missing non-white patients differed greatly to the non-white patients who responded, it is likely the lack of association would remain (see Additional file 1: supplementary information).

\section{Statistical analyses}

All analyses were carried out using SPSS 20. Patient characteristics between groups were compared using ANOVAs, chi-square tests and Kruskal-Wallis tests using conventional two-sided $5 \%$ significance level. Appropriate adjustments - Scheffé adjustments for pairwise differences in ANOVA, Bonferroni adjustments in z-tests of category proportions and Mann-Whitney $U$ tests - were made to account for multiple testing when carrying out pairwise comparisons. Cronbach's alpha was used to assess the internal consistency of the two API subscales in the ESRD group. A confounder-adjusted analysis has been carried out for all variables considered in the study in accordance with the definition of a potential confounder[23]. Variables from this analysis were also used to inform the choice of variables in the multivariable regression model. The multivariable linear regression with a backwards step-wise selection method was used to identify the variables that are associated with the API decision-making preference of patients. The same selection method was used for API information seeking. Variables with $\mathrm{p}$-value of less than 0.15 in the single variable analysis were considered for selection in the multivariable analysis. In the multivariable analysis, three variables were considered clinically important: age, education and group. The other variables were removed from the model until only those with a p-value less than 0.05 remained. A linear mixed effects model with centre as the random effect has been used to account for a possible centre effect .The ICC (intraclass correlation coefficient) is the measure of the ratio of the between cluster variance to the total variance (between-cluster + withincluster). ICC close to 1 indicates the people in the cluster are very similar, whereas ICC close to 0 indicates the between-cluster variability is small compared to the within-cluster variability. Three patient subgroups, based on the API decision-making scores were also constructed, to understand factors associated with these scores in the highest and lowest tertiles. The patient subgroup characteristics were examined using Mann-Whitney U tests, Fisher's exact tests and linear-by-linear chi-square tests. Paired t-tests were used to examine change over time for the prospective data from 30 Group 'A' (CKD-5) patients.

\section{Results}

\section{Demographic and clinical characteristics of the ESRD} population

A total of 458 responses were available. $39.7 \%$ of the responses came from predialysis patients. Overall, patients receiving home HD were younger, more educated and in employment. They had the support of care-givers at home, predominantly, spouses. There were significantly greater numbers of patients with diabetes and greater comorbidity burden in the 'predialysis' and 'in-centre' dialysis groups. There was no significant difference between groups with respect to previously diagnosed affective disorders or in their screening for anxiety and depression using validated inventories. These group comparisons are important to adjust later analyses for potential confounders. The differences between the groups are illustrated in Table 1. Due to clinical 
Table 1 Demographic, clinical and psychosocial characteristics of the ESRD study population

\begin{tabular}{|c|c|c|c|c|c|c|}
\hline Variable & & Total $(n=458)$ & Predialysis(A) $(n=182)$ & In-centre $\mathrm{HD}(\mathrm{B})(n=186)$ & Home $\mathrm{HD}(C)(n=90)$ & $p$-value \\
\hline Age Mean (std. dev.) & & & $59.84(13.28)$ & $56.84(14.78)$ & $51.81(11.67)$ & $\begin{array}{l}p<0.001^{\mathrm{a}} \mathrm{A}>\mathrm{C} \\
p<0.001^{\mathrm{b}} \mathrm{B}>\mathrm{C} \\
p=0.017^{\mathrm{b}}\end{array}$ \\
\hline Gender & Male & 296 (64.6\%) & $110(60.4 \%)$ & 119 (64.0\%) & $67(74.4 \%)$ & $p=0.073^{c}$ \\
\hline Education & Post high school education & $111(25.1 \%)$ & $43(24.2 \%)$ & $31(17.5 \%)$ & $37(42.5 \%)$ & $\begin{array}{l}p<0.001^{c} \\
A<C p<0.05^{d} \\
B<C p<0.05^{d}\end{array}$ \\
\hline \multirow[t]{4}{*}{ Employment } & Retired & $213(46.8 \%)$ & $94(51.6 \%)$ & $87(47.3 \%)$ & $32(36.0 \%)$ & $\begin{array}{l}p<0.001^{c} \\
\text { Retired }\end{array}$ \\
\hline & Unemployed & $115(25.3 \%)$ & $31(17.0 \%)$ & $64(34.8 \%)$ & $20(22.5 \%)$ & $\begin{array}{l}\text { A }>C p<0.05^{d} \\
\text { Unemployed }\end{array}$ \\
\hline & Self-employed & $35(7.7 \%)$ & $17(9.3 \%)$ & $10(5.4 \%)$ & $8(9.0 \%)$ & $\mathrm{A}<\mathrm{B} p<0.05^{\mathrm{d}}$ \\
\hline & Salaried & $92(20.2 \%)$ & $40(22.0 \%)$ & $23(12.5 \%)$ & $29(32.6 \%)$ & $\begin{array}{l}\text { Salaried } \\
A>B p<0.05^{d} \\
C>B p<0.05^{d}\end{array}$ \\
\hline Ethnicity & Non-white & $46(10.1 \%)$ & $14(7.7 \%)$ & $20(10.8 \%)$ & $12(13.3 \%)$ & $p=0.32^{c}$ \\
\hline $\begin{array}{l}\mathrm{BMI}\left(\mathrm{kg} / \mathrm{m}^{\mathrm{b}} \text { ) Median (interquartile }\right. \\
\text { range) }\end{array}$ & & & $28.38(24.27,32.45)$ & $26.50(23.08,31.51)$ & $26.53(23.63,30.83)$ & $\begin{array}{l}p=0.031^{\mathrm{e}} \\
A>B p=0.040^{f} \\
A>C p=0.21^{f} \\
C>B p=1.00^{f}\end{array}$ \\
\hline \multirow[t]{3}{*}{ Smoking status } & Never smoked & $257(56.7 \%)$ & 98 (54.1\%) & $103(56.6 \%)$ & $56(62.2 \%)$ & \multirow[t]{3}{*}{$p=0.57^{c}$} \\
\hline & Ex-smoker & $133(29.4 \%)$ & $54(29.8 \%)$ & $53(29.1 \%)$ & $26(28.9 \%)$ & \\
\hline & Current & $63(13.9 \%)$ & $29(16.0 \%)$ & $26(14.3 \%)$ & $8(8.9 \%)$ & \\
\hline \multirow[t]{5}{*}{ Caregiver } & Spouse/partner & $250(56.2 \%)$ & $111(62.0 \%)$ & $83(46.6 \%)$ & $56(63.6 \%)$ & $\begin{array}{l}p=0.004^{c} \\
\text { Spouse/part }\end{array}$ \\
\hline & Child carer & $24(5.4 \%)$ & $12(6.7 \%)$ & $6(3.4 \%)$ & $6(6.8 \%)$ & $\begin{array}{l}A>B p<0.05^{d} \\
C>B p<0.05^{d}\end{array}$ \\
\hline & Parent carer & $34(7.6 \%)$ & $10(5.6 \%)$ & $16(9.0 \%)$ & 8 (9.1\%) & $F, R, S$ or $C$ \\
\hline & Friend, relative, sibling or carer & $19(4.3 \%)$ & $3(1.7 \%)$ & $13(7.3 \%)$ & $3(3.4 \%)$ & $\mathrm{B}>\mathrm{A} p<0.05^{\mathrm{d}}$ \\
\hline & Alone & $118(26.5 \%)$ & $43(24.0 \%)$ & 60 (33.7\%) & 15 (17.0\%) & $\begin{array}{l}\text { Alone } \\
\text { B }>\text { C } p<0.05^{d}\end{array}$ \\
\hline \multirow[t]{5}{*}{ Marital status } & Married & $253(55.2 \%)$ & $106(58.2 \%)$ & 89 (47.8 \%) & $58(64.4 \%)$ & \multirow{2}{*}{$\begin{array}{l}p=0.020^{c} \\
\text { Married } \\
B<C p<0.05^{d}\end{array}$} \\
\hline & Partner & $27(5.9 \%)$ & $11(6.0 \%)$ & $10(5.4 \%)$ & $6(6.7 \%)$ & \\
\hline & Single & $103(22.5 \%)$ & $38(20.9 \%)$ & $54(29.0 \%)$ & $11(12.2 \%)$ & \multirow{3}{*}{$\begin{array}{l}\text { Single } \\
B>C p<0.05^{d}\end{array}$} \\
\hline & Divorced or separated & $40(8.7 \%)$ & $11(6.0 \%)$ & $17(9.1 \%)$ & $12(13.3 \%)$ & \\
\hline & Widowed & $35(7.6 \%)$ & $16(8.8 \%)$ & $16(8.6 \%)$ & $3(3.3 \%)$ & \\
\hline Psych usage & Never offered & 180 (40.8 \%) & 70 (38.9 \%) & 84 (48.8 \%) & 26 (29.2 \%) & \\
\hline
\end{tabular}


Table 1 Demographic, clinical and psychosocial characteristics of the ESRD study population (Continued)

\begin{tabular}{|c|c|c|c|c|c|c|}
\hline & Never used & $209(47.4 \%)$ & $98(54.4 \%)$ & $69(40.1 \%)$ & $42(47.2 \%)$ & $P<0.001^{c}$ \\
\hline & Used and found useful & 36 (8.2 \%) & $6(3.3 \%)$ & $16(9.3 \%)$ & $14(15.7 \%)$ & $\begin{array}{l}\text { Never offered } \\
B>C p<0.05^{d}\end{array}$ \\
\hline & Used but not useful & $16(3.6 \%)$ & $6(3.3 \%)$ & $3(1.7 \%)$ & 7 (7.9 \%) & $\begin{array}{l}\text { Never used } \\
\text { A }>B \quad p<0.05^{d} \\
\text { Used and found useful } \\
C>A p<0.05^{d} \\
\text { Used but not useful } \\
C>B \quad p<0.05^{d}\end{array}$ \\
\hline \multirow[t]{9}{*}{ Primary cause of ESRD } & Hypertensive Nephrosclerosis & $55(12.0 \%)$ & $34(18.7 \%)$ & $12(6.5 \%)$ & $9(10.0 \%)$ & $\begin{array}{l}p<0.001^{c} \\
\text { Hyp Neph } \\
\text { A }>B \quad p<0.05^{d}\end{array}$ \\
\hline & Diabetic Nephropathy & $96(21.0 \%)$ & $45(24.7 \%)$ & $42(22.6 \%)$ & $9(10.0 \%)$ & $\begin{array}{l}\text { Diab Neph } \\
A>C p<0.05^{d} \\
B>C p<0.05^{d}\end{array}$ \\
\hline & Glomerulonephritis & $65(14.2 \%)$ & $19(10.4 \%)$ & $30(16.1 \%)$ & $16(17.8 \%)$ & \multirow{7}{*}{$\begin{array}{l}\text { Polycystic KD } \\
C>A p<0.05^{d} \\
C>B p<0.05^{d}\end{array}$} \\
\hline & Polycystic Kidney Disease & $55(12.0 \%)$ & 18 (9.9 \%) & 18 (9.7%) & $19(21.1 \%)$ & \\
\hline & Renovascular Disease & $12(2.6 \%)$ & $4(2.2 \%)$ & $8(4.3 \%)$ & $0(0 \%)$ & \\
\hline & $\begin{array}{l}\text { Chronic Pyelonephritis/ Reflux } \\
\text { Nephropathy }\end{array}$ & $29(6.3 \%)$ & $8(4.4 \%)$ & $15(8.1 \%)$ & $6(6.7 \%)$ & \\
\hline & Others & $83(18.1 \%)$ & $36(19.8 \%)$ & $32(17.2 \%)$ & $15(16.7 \%)$ & \\
\hline & Unknown & $63(13.8 \%)$ & $18(9.9 \%)$ & $29(15.6 \%)$ & $16(17.8 \%)$ & \\
\hline & Yes & $\begin{array}{l}121 / 276 \\
(43.8 \%)\end{array}$ & $\sim$ & $83(44.6 \%)$ & $38(42.2 \%)$ & \\
\hline Hypertension & Yes & $348(76.0 \%)$ & $152(83.5 \%)$ & $123(66.1 \%)$ & $73(81.1 \%)$ & $\begin{array}{l}p<0.001^{C} \\
A>B p<0.05^{d} \\
C>B p<0.05^{d}\end{array}$ \\
\hline Diabetes & Yes & $123(27.0 \%)$ & $56(30.8 \%)$ & $56(30.4 \%)$ & $11(12.4 \%)$ & $\begin{array}{l}p=0.002^{c} \\
A>C p<0.05^{d} \\
B>C p<0.05^{d}\end{array}$ \\
\hline H/O Anxiety & Yes & $14(3.1 \%)$ & $5(2.7 \%)$ & $5(2.7 \%)$ & $4(4.4 \%)$ & $p=0.69^{c}$ \\
\hline H/O Depression & Yes & $48(10.5 \%)$ & $17(9.3 \%)$ & $18(9.7 \%)$ & $13(14.4 \%)$ & $p=0.39^{c}$ \\
\hline CCI Median (inter-quartile range) & & & $5.00(3.75,6.00)$ & $4.00(3.00,6.00)$ & $3.50(2.00,5.00)$ & $\begin{array}{l}p<0.001^{\mathrm{e}} \\
\mathrm{A}>\mathrm{B} p=0.60^{\mathrm{f}} \\
\mathrm{A}>\mathrm{C} \bar{p}<0.001^{\mathrm{f}} \\
\mathrm{B}>\mathrm{C} p=0.004^{\mathrm{f}}\end{array}$ \\
\hline \multirow[t]{5}{*}{ BDI } & $0-10$ & $239(52.2 \%)$ & $102(56.0 \%)$ & $91(48.9 \%)$ & $46(51.1 \%)$ & \multirow[t]{5}{*}{$p=0.783$} \\
\hline & $11-15$ & $73(15.9 \%)$ & $27(14.8 \%)$ & $31(16.7 \%)$ & $15(16.7 \%)$ & \\
\hline & $16-20$ & $45(9.8 \%)$ & $18(9.9 \%)$ & $20(10.8 \%)$ & $7(7.8 \%)$ & \\
\hline & $21-25$ & $44(9.6 \%)$ & $17(9.3 \%)$ & $20(10.8 \%)$ & $7(7.8 \%)$ & \\
\hline & $26-30$ & 25 (5.5 \%) & 6 (3.3\%) & 13 (7.0 \%) & 6 (6.7\%) & \\
\hline
\end{tabular}


Table 1 Demographic, clinical and psychosocial characteristics of the ESRD study population (Continued)

\begin{tabular}{|c|c|c|c|c|c|c|}
\hline & $\geq 31$ & $32(7.0 \%)$ & $12(6.6 \%)$ & $11(5.9 \%)$ & $9(10.0 \%)$ & \\
\hline \multirow[t]{4}{*}{ STAI State } & $20-29$ & $148(33.4 \%)$ & $54(30.0 \%)$ & $61(34.5 \%)$ & $33(38.4 \%)$ & \multirow[t]{4}{*}{$p=0.863$} \\
\hline & $30-39$ & $131(29.6 \%)$ & $57(31.7 \%)$ & $53(29.9 \%)$ & $21(24.4 \%)$ & \\
\hline & $40-49$ & $100(22.6 \%)$ & $42(23.3 \%)$ & $38(21.5 \%)$ & $20(23.3 \%)$ & \\
\hline & $\geq 50$ & $64(14.4 \%)$ & $27(15.0 \%)$ & $25(14.1 \%)$ & $12(14.0 \%)$ & \\
\hline \multirow[t]{4}{*}{ STAI Trait } & $20-29$ & $121(27.6 \%)$ & $47(26.3 \%)$ & $49(28.2 \%)$ & $25(29.1 \%)$ & \multirow[t]{4}{*}{$p=0.433$} \\
\hline & $30-39$ & $124(28.2 \%)$ & $46(25.7 \%)$ & $54(31.0 \%)$ & $24(27.9 \%)$ & \\
\hline & $40-49$ & $111(25.3 \%)$ & $55(30.7 \%)$ & $39(22.4 \%)$ & $17(19.8 \%)$ & \\
\hline & $\geq 50$ & $83(18.9 \%)$ & $31(17.3 \%)$ & $32(18.4 \%)$ & $20(23.3 \%)$ & \\
\hline \multicolumn{2}{|c|}{ TMT A Median (inter-quartile Range) } & & $46.65(32.25,60.00)$ & $47.00(36.00,69.00)$ & $35.50(30.00,47.53)$ & $\begin{array}{l}p<0.001^{\mathrm{e}} \\
\mathrm{B}>\mathrm{A} p=0.39^{\mathrm{f}} \\
\mathrm{A}>\mathrm{C} p=0.001^{\mathrm{f}} \\
\mathrm{B}>\mathrm{C} p<0.001^{\mathrm{f}}\end{array}$ \\
\hline \multicolumn{2}{|c|}{ TMT B Median (inter-quartile range) } & & $90.00(68.50,120.00)$ & $113.00(73.00,145.00)$ & $74.00(61.00,94.00)$ & $\begin{array}{l}p<0.0015 \\
B>A p=0.0236 \\
A>C p=0.0076 \\
B>C p<0.0016\end{array}$ \\
\hline \multirow[t]{5}{*}{$3 \mathrm{MS}$} & $\leq 75$ & $11(2.6 \%)$ & $2(1.2 \%)$ & 8 (4.6 \%) & $1(1.3 \%)$ & \multirow[t]{5}{*}{$p=0.0517$} \\
\hline & $76-80$ & $14(3.3 \%)$ & $6(3.5 \%)$ & $6(3.5 \%)$ & $2(2.6 \%)$ & \\
\hline & $81-85$ & 35 (8.3 \%) & $9(5.3 \%)$ & 21 (12.1\%) & $5(6.5 \%)$ & \\
\hline & $86-93$ & $157(37.3 \%)$ & 64 (37.4\%) & 69 (39.9\%) & 24 (31.2 \%) & \\
\hline & $94-100$ & 204 (48.5 \%) & 90 (52.6 \%) & 69 (39.9\%) & 45 (58.4 \%) & \\
\hline
\end{tabular}

aANOVA $p$-value for overall between groups mean differences

${ }^{b}$ Scheffe adjusted $p$-values for comparison of pair-wise group means

'Pearson Chi-Square $p$-value

${ }^{d}$-test comparing category proportions between groups, $p$-value with Bonferroni adjustment for multiple testing

eKruskal-Wallis test $p$-value

${ }^{f}$ Mann-Whitney $U$ test $p$-value with adjustment for multiple testing 
importance and due to the fact there were differences between the groups, it was automatically included as a variable in both the API-DM and API-IS analyses. The group variable being included should therefore account for the differences in characteristics of the group.

\section{API in ESRD population}

We measured the internal consistency of the items in the API, in our study population using Cronbach's alpha. This was acceptable for both information-seeking (Cronbach's alpha $=0.774$ ) and decision-making (Cronbach's Alpha $=0.714$ ) subscales of the API. The mean and standard deviations for all the items in both subscales are presented in the Additional file 2: supplementary material.

\section{Descriptive data analysis of the two subscales and clinical vignettes}

The median score for the API information-seeking scale and the API decision-making scale in all three study cohorts is depicted in box plots (Fig. 2). For the API clinical vignettes, worsening symptom severity was associated with a change in treatment decision-making preference scores, and most patients in the collective ESRD group wanted shared decision-making with their healthcare provider if symptoms hypothetically worsened (Fig. 3).

\section{Demographic, clinical and psychosocial factors predicting decision-making in ESRD}

In the single variable analysis of the information-seeking subscale scores, the predictors at the $15 \%$ significance level of high IS scores were (linear regression with just the variable of interest in the model): age, education, study group, gender, marital status, heart failure, BDI score, 3MS, IMD score (index of multiple deprivation), first choice of dialysis modality and perceived ability to consider self-cannulation for haemodialysis.

In the single variable analysis of the decision-making subscale (Table 2), variables significant at the $5 \%$ level (a linear regression with just the variable of interest in the model) and were considered for the multivariable analysis include: age, study group, employment, marital status, psychology service use, diabetes, heart failure, ischaemic heart disease history, CCI, TMT A and API information-seeking subscale score. Additionally, variables up to $15 \%$ significance were also included in the multivariable model. These include gender, ethnicity, informal caregiver availability and patient attendance of a treatment options education session. The confounder adjusted analysis has highlighted a number of significant variables in common with the unadjusted single variable analysis. The multiple linear regression selection process for the decision-making subscale (Table 3), which had

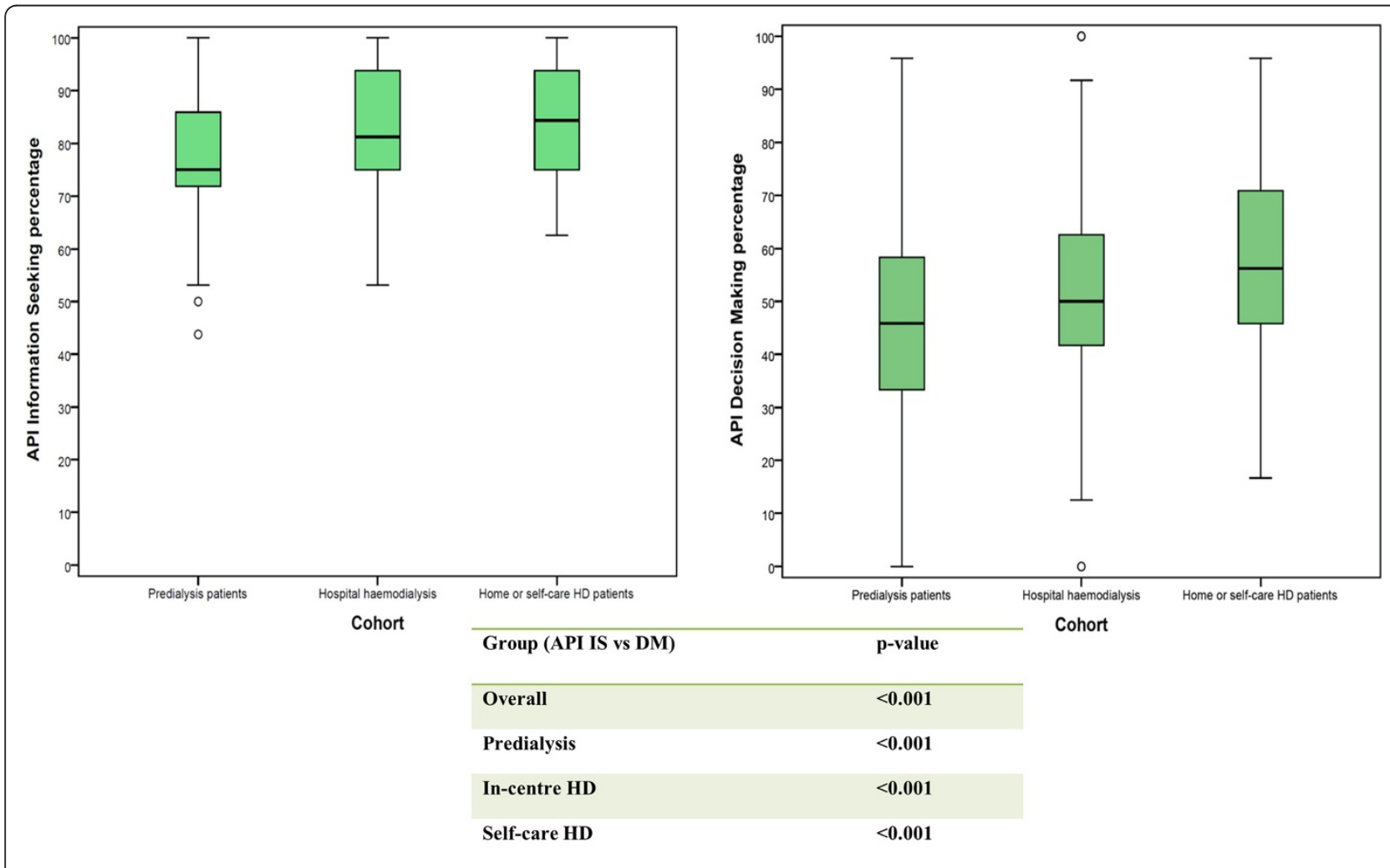

Fig. 2 Box Plots showing the median scores on the API for Information-seeking and Decision-making subscales in all three study groups 


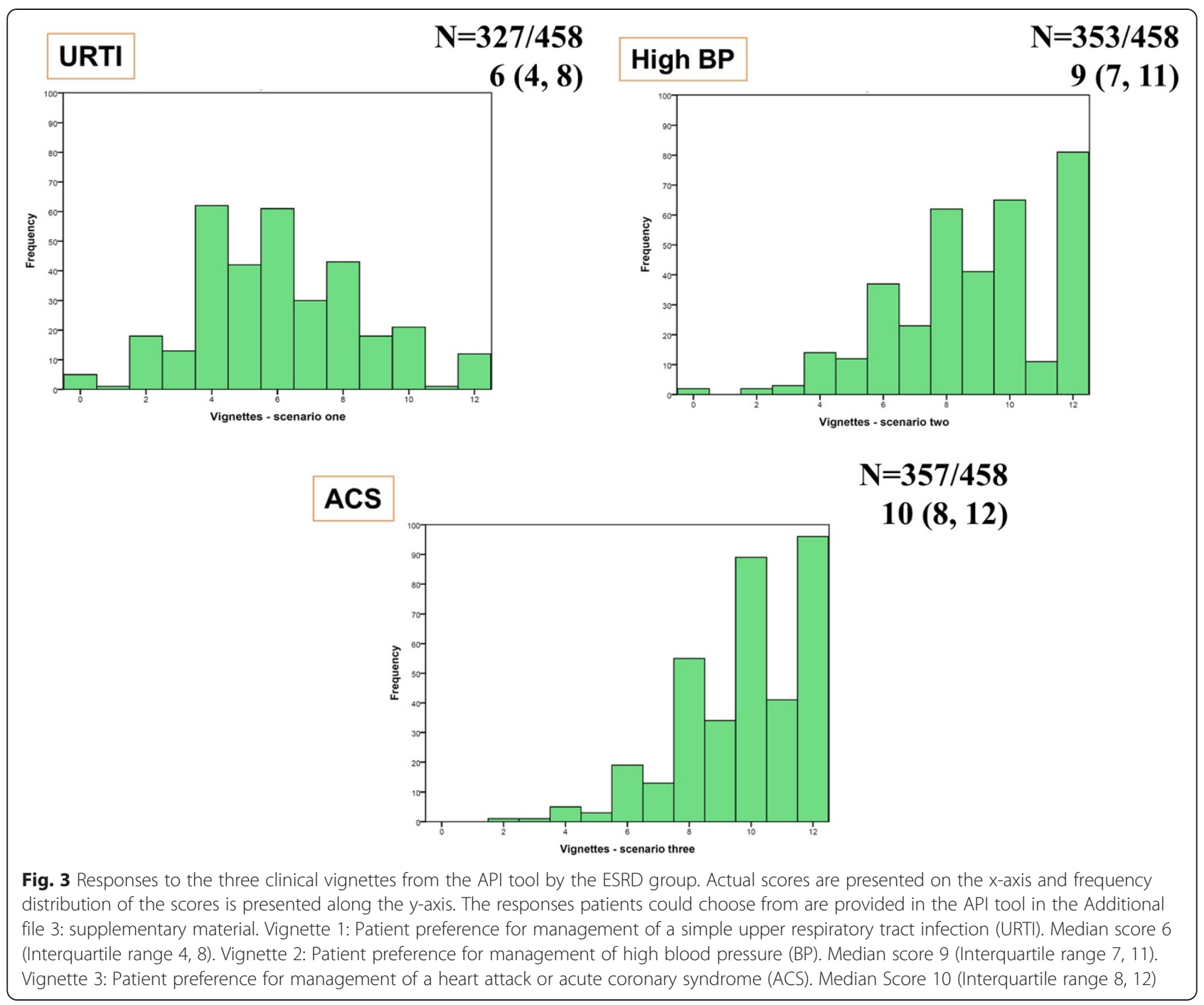

age, education and study group adjusted for, selected lower age, female gender, marital status, API information-seeking scores and white ethnicity background at $5 \%$ significance level, in favour of greater autonomy in decision-making. Education was not significant in the multivariable analysis. The multiple linear regression selection process for the information-seeking scores (Table 3), which had age, education and study group adjusted for, selected lower age, post high school education, marital status and per category increase in BDI score at the $5 \%$ significance level as significantly associated with information-seeking. In the model where 'centre-effect' was evaluated, sensitivity analysis that suggests very little change for API-DM and for API-IS education and age are slightly less significant than in the model without the centre-effect included. The likelihood ratio test in both cases failed to reject the null hypothesis of there being no difference between the mixed effects model and the ordinary linear model.

\section{'Autonomists' vs 'Delegators'}

Decision-making subscale scores were subdivided into tertiles to identify variables associated with high (DM > 70: empirically designated as autonomists) and low DM ( $\leq 30$ : empirically designated as delegators) scores (Fig. 4). This shows association of higher decision-making scores with lower age, lower comorbidity index scoring, higher executive brain function, belonging in the selfcaring cohort and being unemployed (although lack of employment may have been a conscious decision of the study participants) (Table 4). Some of these variables separate the two patient clusters (e.g. CCI, higher cognitive scores etc.), but these have not featured in the final multivariable model involving the total patient cohort, possibly 
Table 2 Single variable analysis and confounder adjusted analysis (Decision-making)

\begin{tabular}{|c|c|c|c|c|c|c|}
\hline \multicolumn{4}{|c|}{ Single Variable Analysis (API-DM) } & \multicolumn{3}{|l|}{ Confounder Adjusted Analysis (API-DM) } \\
\hline \multicolumn{2}{|c|}{ Variable of interest } & $\mathrm{EMM}^{*}(95 \% \mathrm{Cl})$ & $\begin{array}{l}p- \\
\text { value }\end{array}$ & Confounders & Regression Coefficient (95 \% Cl) & $\begin{array}{l}p- \\
\text { value }\end{array}$ \\
\hline \multicolumn{2}{|l|}{ Age $\left(\right.$ per year) ${ }^{* *}$} & $71.60-0.37(-0.48,-0.26)$ & $<0.001$ & $\begin{array}{l}\text { Ethnicity, Caregiver, Marital status, Education session, } \\
\text { Psych service use, API-IS, group }\end{array}$ & $-0.33(-0.48,-0.19)$ & $<0.001$ \\
\hline \multirow[t]{2}{*}{ Education } & High school & $49.88(47.96,51.80)$ & \multirow[t]{2}{*}{0.29} & \multirow{2}{*}{$\begin{array}{l}\text { Age, ethnicity, psych service use, diabetes, education } \\
\text { session }\end{array}$} & \multirow[t]{2}{*}{$-2.31(-6.12,1.50)$} & \multirow[t]{2}{*}{0.23} \\
\hline & Post high school & $51.91(48.64,55.19)$ & & & & \\
\hline \multirow[t]{2}{*}{ Gender } & Male & $49.49(47.44,51.53)$ & \multirow[t]{2}{*}{0.092} & \multirow[t]{2}{*}{$\mathrm{CCl}$} & \multirow[t]{2}{*}{$-2.03(-5.47,1.41)$} & \multirow[t]{2}{*}{0.25} \\
\hline & Female & $52.45(49.67,55.24)$ & & & & \\
\hline \multirow[t]{5}{*}{ Employment } & Retired & $46.05(43.69,48.42)$ & \multirow[t]{5}{*}{$<0.001$} & \multirow{5}{*}{$\begin{array}{l}\text { Ethnicity, caregiver, marital status, psych service use, } \\
\text { CCl, education session, TMT A }\end{array}$} & \multirow{5}{*}{$\begin{array}{l}-2.61(-7.65,2.42) 7.97(2.63,13.30) 0.04 \\
(-7.47,7.55)\end{array}$} & \multirow[t]{5}{*}{0.001} \\
\hline & Unemployed & $57.13(53.94,60.32)$ & & & & \\
\hline & Self employed & $50.00(44.25,55.75)$ & & & & \\
\hline & Salaried & $56.63(49.08,56.18)$ & & & & \\
\hline & Employed & $51.90(48.79,55.02)$ & & & & \\
\hline \multirow[t]{2}{*}{ Ethnicity } & White & $50.90(49.16,52.64)$ & \multirow[t]{2}{*}{0.13} & \multirow[t]{2}{*}{ Age, employment } & \multirow[t]{2}{*}{$8.22(2.86,13.59)$} & \multirow[t]{2}{*}{0.003} \\
\hline & Non-white & $46.65(41.50,51.80)$ & & & & \\
\hline \multirow[t]{3}{*}{ BMl } & $<25$ & $49.89(47.08,52.71)$ & \multirow[t]{3}{*}{0.74} & \multirow[t]{3}{*}{ Age, gender } & \multirow[t]{3}{*}{$-2.58(-6.42,1.26)-0.06(-4.05,3.93)$} & \multirow[t]{3}{*}{0.33} \\
\hline & 25-29.99 & $50.12(47.14,53.09)$ & & & & \\
\hline & $\geq 30$ & $51.37(48.52,54.23)$ & & & & \\
\hline \multirow[t]{3}{*}{ Smoking Status } & Never smoked & $51.19(48.97,53.40)$ & 0.65 & Age, gender, employment & $1.98(-2.81,6.76) 2.50(-2.78,7.79)$ & 0.64 \\
\hline & Ex-smoker & $49.39(46.30,52.48)$ & & & & \\
\hline & Current & $50.33(45.90,54.77)$ & & & & \\
\hline Caregiver & Spouse or partner & $50.31(48.07,52.54)$ & 0.12 & Employment, psych service use, $\mathrm{CCl}$, group & $3.56(-0.44,7.55)-2.08(-9.89,5.72) 1.66$ & 0.33 \\
\hline & Child & $46.56(39.26,53.86)$ & & & $(-5.55,8.88) 3.28(-5.15,11 . / 1)$ & \\
\hline & Parent & $57.35(51.35,63.36)$ & & & & \\
\hline & $\begin{array}{l}\text { Friend, RelativeSibling, } \\
\text { Carer }\end{array}$ & $53.47(45.22,61.72)$ & & & & \\
\hline & Alone & $49.05(45.82,52.27)$ & & & & \\
\hline Marital Status & Married or partner & $51.22(49.12,53.31)$ & 0.006 & Age, employment, psych service use,TMT A, group & $7.88(1.51,14.25) 3.95(-3.40,11.31) 4.29$ & 0.041 \\
\hline & Single & $51.86(48.44,55.28)$ & & & & \\
\hline & Divorced or separated & $51.28(45.73,56.84)$ & & & & \\
\hline & Widowed & $40.36(34.49,46.22)$ & & & & \\
\hline Psych service use & Not used & $49.75(47.96,51.54)$ & 0.088 & Age, employment, caregiver, marital status, & $0.56(-5.13,6.24)$ & 0.85 \\
\hline & Used & $54.25(49.40,59.09)$ & & & & \\
\hline Diabetes & No & $51.63(49.70,53.55)$ & 0.021 & Age, employment, psych service use & $2.75(-0.95,6.46)$ & 0.14 \\
\hline
\end{tabular}


Table 2 Single variable analysis and confounder adjusted analysis (Decision-making) (Continued)

\begin{tabular}{|c|c|c|c|c|c|c|}
\hline & Yes & $47.21(43.99,50.43)$ & & & & \\
\hline \multirow[t]{2}{*}{ Heart Failure } & No & $50.91(49.23,52.60)$ & \multirow[t]{2}{*}{0.042} & \multirow[t]{2}{*}{ Age } & \multirow[t]{2}{*}{$5.61(-1.76,12.99)$} & \multirow[t]{2}{*}{0.14} \\
\hline & Yes & $42.99(35.54,50.44)$ & & & & \\
\hline \multirow[t]{2}{*}{$\mathrm{IHD}$} & No & $51.51(49.64,53.39)$ & \multirow[t]{2}{*}{0.032} & \multirow[t]{2}{*}{ Age, gender, employment, diabetes, TMT A } & \multirow[t]{2}{*}{$-0.50(-4.68,3.68)$} & \multirow[t]{2}{*}{0.81} \\
\hline & Yes & $47.24(43.81,50.66)$ & & & & \\
\hline \multirow[t]{2}{*}{ Stroke } & No & $50.79(49.07,52.51)$ & \multirow[t]{2}{*}{0.28} & \multirow[t]{2}{*}{ Age, IHD, education session } & \multirow[t]{2}{*}{$-0.05(-6.13,6.04)$} & \multirow[t]{2}{*}{0.99} \\
\hline & Yes & $47.38(41.46,53.31)$ & & & & \\
\hline \multirow{2}{*}{$\begin{array}{l}\text { Solid Organ } \\
\text { Malignancy }\end{array}$} & No & $50.26(48.51,52.01)$ & \multirow[t]{2}{*}{0.36} & \multirow{2}{*}{$\begin{array}{l}\text { Age, employment, caregiver, psych service use, } \\
\text { diabetes, group }\end{array}$} & \multirow[t]{2}{*}{$-2.71(-8.09,2.67)$} & \multirow[t]{2}{*}{0.32} \\
\hline & Yes & $52.78(47.72,57.84)$ & & & & \\
\hline \multicolumn{2}{|c|}{ Charlson Comorbidity Index (per unit)** } & $60.67-2.26(-3.07,-1.44)$ & $<0.001$ & $\begin{array}{l}\text { Gender, ethnicity, caregiver, marital status, psych } \\
\text { service use, education session }\end{array}$ & $-1.87(-2.79,-0.96)$ & $<0.001$ \\
\hline \multicolumn{2}{|c|}{$\begin{array}{l}\text { BDI in } 6 \text { categories(per category) -low } \\
\text { score to high score** }\end{array}$} & $49.200 .60(-0.43,1.63)$ & 0.25 & Age, employment, diabetes, API IS & $-0.28(-1.33,0.77)$ & 0.61 \\
\hline \multicolumn{2}{|c|}{$\begin{array}{l}\text { Anxiety State in } 4 \text { categories(per category) } \\
\text {-low score to high score** }\end{array}$} & $49.560 .47(-1.14,2.08)$ & 0.56 & Age, employment, ethnicity, marital status & $-0.35(-1.92,1.22)$ & 0.66 \\
\hline \multicolumn{2}{|c|}{$\begin{array}{l}\text { Anxiety Trait in } 4 \text { categories(per category) - } \\
\text { low score to high score }\end{array}$} & $48.550 .82(-0.75,2.38)$ & 0.31 & Employment,ethnicity, marital status,CCI, API IS & $-0.60(-2.18,0.98)$ & 0.46 \\
\hline \multicolumn{2}{|c|}{$\begin{array}{l}\text { 3MS in } 5 \text { categories(per category) -high } \\
\text { score to low score** }\end{array}$} & $51.64-0.63(-2.49,1.22)$ & 0.50 & Age, employment, diabetes, IHD, TMT A & $0.98(-1.03,2.99)$ & 0.31 \\
\hline \multirow{2}{*}{$\begin{array}{l}\text { Options education } \\
\text { session }\end{array}$} & No & $51.29(49.40,53.18)$ & \multirow[t]{2}{*}{0.11} & \multirow[t]{2}{*}{ Age, employment, group } & \multirow[t]{2}{*}{$0.11(-3.73,3.96)$} & \multirow[t]{2}{*}{0.95} \\
\hline & Yes & $48.13(44.77,51.48)$ & & & & \\
\hline \multirow{3}{*}{$\begin{array}{l}\text { Predialysis } \\
\text { education } \\
\text { experience }\end{array}$} & $\begin{array}{l}\text { Very poor/Not useful/ } \\
\text { Inadequate }\end{array}$ & $52.53(46.56,58.49)$ & \multirow[t]{3}{*}{0.18} & \multirow[t]{3}{*}{$\begin{array}{l}\text { Employment, caregiver, marital status, psych service } \\
\text { use, education session, group }\end{array}$} & \multirow[t]{3}{*}{$1.95(-4.91,8.80) 1.58(-2.30,5.45)$} & 0.69 \\
\hline & Good & $50.72(48.03,53.40)$ & & & & \\
\hline & Excellent & $47.62(44.79,50.45)$ & & & & \\
\hline TMT A (per unit)** & & $54.21-0.07(-0.13,-0.01)$ & 0.018 & Age, marital status, psych service use, diabetes, IHD & $-0.01(-0.07,0.05)$ & 0.78 \\
\hline TMT B (per unit)** & & $54.08-0.02(-0.06,0.01)$ & 0.22 & $\begin{array}{l}\text { Age, ethnicity, marital status, psych service use, } \\
\text { diabetes,IHD, TMT A }\end{array}$ & - & - \\
\hline API IS & $\begin{array}{l}\text { Per percentage } \\
\text { increase }\end{array}$ & $26.560 .30(0.15,0.44)$ & $<0.001$ & Age, group & $0.19(0.04,0.33)$ & 0.010 \\
\hline Group & $\begin{array}{l}\text { PredialysisHospitalSelf- } \\
\text { care }\end{array}$ & $\begin{array}{l}45.88(43.32,48.44) 51.96(49.43 \\
54.48) 56.81(53.20,60.41)\end{array}$ & $<0.001$ & $\begin{array}{l}\text { Age, caregiver, marital status, psych service use, } \\
\text { diabetes, education session, TMT A, API IS }\end{array}$ & $-7.09(-12.09,-2.09)-1.76(-6.82,3.29)$ & 0.006 \\
\hline
\end{tabular}

*Estimated Marginal Mean

**The results for these continuous variables are presented as the intercept, the parameter estimate and the $95 \% \mathrm{Cl}$ of the parameter estimate

$\Delta$ Analysis not reported as $>25 \%$ missing values in the dataset 
Table 3 Multivariable Analysis (Age, education and group included a priori)

\begin{tabular}{|c|c|c|c|}
\hline \multicolumn{4}{|c|}{ MULTIVARIABLE LINEAR REGRESSION ANALYSIS: DECISION-MAKING SUBSCALE } \\
\hline Variable & & Parameter estimate $(95 \% \mathrm{Cl})$ & $p$-value \\
\hline \multirow{2}{*}{ Education } & High school & $-1.23(-4.83,2.36)$ & 0.50 \\
\hline & Post high school & $\sim$ & \\
\hline \multirow[t]{3}{*}{ Group } & Predialysis & $-8.20(-12.59,-3.81)$ & $<0.001$ \\
\hline & In-centre HD & $-1.68(-6.05,2.68)$ & \\
\hline & Home HD & $\sim$ & \\
\hline \multirow[t]{2}{*}{ Gender } & Male & $-3.29(-6.52,-0.07)$ & 0.046 \\
\hline & Female & $\sim$ & \\
\hline \multirow[t]{4}{*}{ Marital Status } & Married or Partner & $8.79(2.92,14.65)$ & 0.015 \\
\hline & Single & $5.43(-1.35,12.21)$ & \\
\hline & Divorced/Separated & $6.26(-1.28,13.80)$ & \\
\hline & Widowed & $\sim$ & \\
\hline \multicolumn{2}{|l|}{ Age (per 10 years) } & $-3.27(-4.54,-2.01)$ & $<0.001$ \\
\hline \multirow[t]{2}{*}{ Ethnicity } & White & $10.62(5.36,15.89)$ & $<0.001$ \\
\hline & Non-white & $\sim$ & \\
\hline API (Information Seeking \%) & Per percentage increase & $0.15(0.01,0.30)$ & 0.035 \\
\hline \multicolumn{2}{|l|}{ Between-centre variability } & $1.17 \times 10^{-19}\left(1.05 \times 10^{-36}, 0.01\right)$ & - \\
\hline \multicolumn{2}{|l|}{ Within-centre variability } & $253.25(221.53,289.52)$ & - \\
\hline \multicolumn{2}{|l|}{ Intra Class Coefficient (ICC) ${ }^{* * *}$} & $4.61 \times 10^{-22}(-)^{* *}$ & - \\
\hline \multicolumn{4}{|c|}{ MULTIVARIABLE LINEAR REGRESSION ANALYSIS: INFORMATION-SEEKING SUBSCALE } \\
\hline \multicolumn{2}{|l|}{ Variable } & Parameter estimate $(95 \% \mathrm{Cl})$ & p-value \\
\hline \multirow[t]{2}{*}{ Education } & High school & $-2.06(-4.41,0.28)$ & 0.085 \\
\hline & Post high school & $\sim$ & \\
\hline \multirow[t]{3}{*}{ Cohort } & Predialysis & $-4.16(-6.99,-1.33)$ & $<0.001$ \\
\hline & In-centre HD & $-0.20(-3.05,2.64)$ & \\
\hline & Home HD & $\sim$ & \\
\hline \multirow[t]{4}{*}{ Marital status } & Married or partner & $1.84(-1.96,5.65)$ & 0.002 \\
\hline & Single & $-2.56(-6.96,1.83)$ & \\
\hline & Divorced or sep. & $4.14(-0.75,9.04)$ & \\
\hline & Widowed & $\sim$ & \\
\hline \multicolumn{2}{|l|}{ Age (per 10 years) } & $-0.79(-1.60,0.03)$ & 0.058 \\
\hline \multicolumn{2}{|c|}{ BDI in 6 categories (per category increase)* } & $0.86(0.24,1.49)$ & 0.007 \\
\hline \multicolumn{2}{|l|}{ Between-centre variability } & $1.14(0.08,16.36)$ & - \\
\hline \multicolumn{2}{|l|}{ Within-centre variability } & $107.87(94.40,123.27)$ & - \\
\hline \multicolumn{2}{|l|}{ Intra Class Coefficient (ICC) ${ }^{* * *}$} & $0.01\left(7.28 \times 10^{-4}, 0.13\right)$ & - \\
\hline
\end{tabular}

*BDI categories: 0-10, 11-15, 16-20, 21-25, 26-30, $\geq 31$

**Standard error estimate of ICC very close to 0 so no confidence interval provided

***ICC is a measure of the correlation of observations in the same cluster. ICC close to 1 indicates the people in the cluster are very similar, whereas ICC close to 0 indicates the between cluster variability is small compared to the within cluster variability

due to differences being most extreme at very high and very low scores.

\section{Decision-making in a prospective observation of the subset of dialysis starters}

Complete API data on dialysis starters was available from 30 predialysis patients who commenced dialysis during the follow-up period up to 1 year from study entry. The data was completed at least 3 months after commencement of dialysis. The mean (SD) decision-making percentage score after commencement of dialysis was 37.79 (16.45), which was not significantly different from the predialysis mean (SD) of 40.52(11.73). 


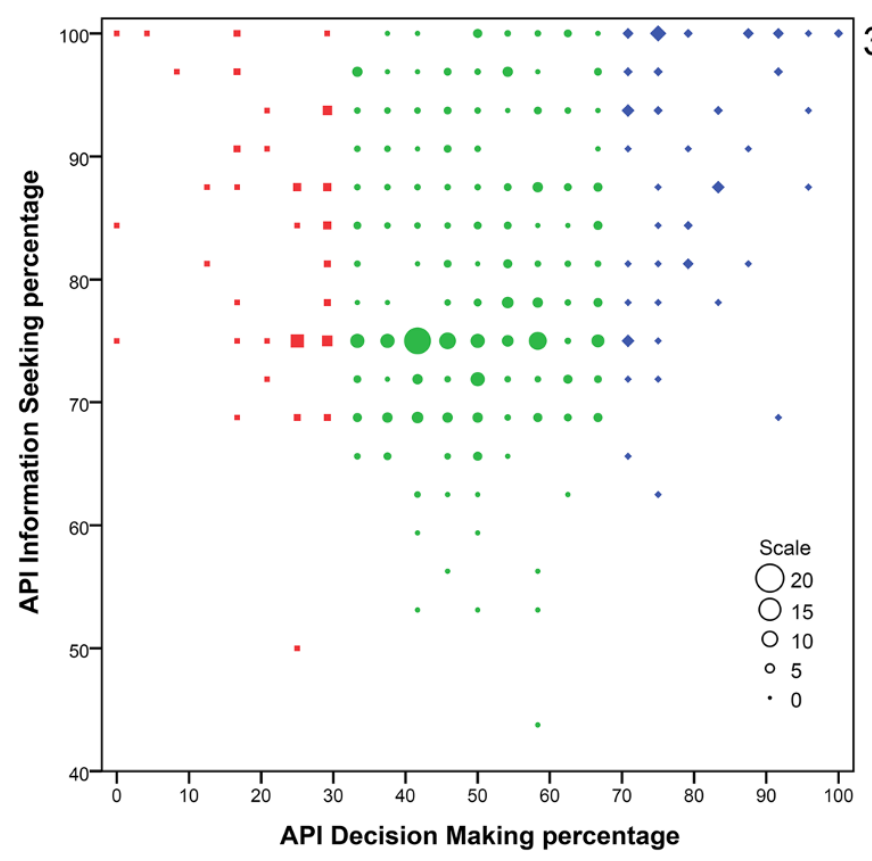

\section{Clusters - clinically determined using decision making percentage \\ - $<30$ \\ $30-70$ \\ $\checkmark>70$}

Fig 4 Distribution of patient clusters determined by high and low decision-making scores

Table 4 Cluster associations with demographic, clinical and psychosocial variables

\begin{tabular}{|c|c|c|c|}
\hline Variable & 1 (Delegators) $(n=57)$ & 3(Autonomists) $(n=66)$ & $p$-value \\
\hline \multicolumn{4}{|l|}{ Group } \\
\hline Predialysis & $30(52.6 \%)$ & $15(22.7 \%)$ & \multirow[t]{3}{*}{$<0.001^{t}$} \\
\hline Hospital & $19(33.3 \%)$ & $27(40.9 \%)$ & \\
\hline Home & $8(14.0 \%)$ & $24(36.4 \%)$ & \\
\hline Age- Median (IQR) & $67.0(56.0-71.5)$ & $52.0(42.8-63.0)$ & $<0.001^{\mathrm{a}}$ \\
\hline \multicolumn{4}{|l|}{ Employment } \\
\hline Retired & $39(68.4 \%)$ & $22(33.3 \%)$ & \multirow[t]{4}{*}{$<0.001^{\mathrm{b}}$} \\
\hline Unemployed & $6(10.5 \%)$ & $24(36.4 \%)$ & \\
\hline Self-employed & $4(7.0 \%)$ & $4(6.1 \%)$ & \\
\hline Salaried & $8(14.0 \%)$ & $16(24.2 \%)$ & \\
\hline \multicolumn{4}{|l|}{ Marital Status } \\
\hline Married or partner & $34(59.6 \%)$ & $43(65.2 \%)$ & \multirow[t]{4}{*}{$0.051^{b}$} \\
\hline Single & $9(15.8 \%)$ & $15(22.7 \%)$ & \\
\hline Divorced or separated & $4(7.0 \%)$ & $6(9.1 \%)$ & \\
\hline Widowed & $10(17.5 \%)$ & $2(3.0 \%)$ & \\
\hline $\begin{array}{l}\mathrm{CCl}^{*} \\
\text { Median (IQR) }\end{array}$ & $\begin{array}{l}n=56 \\
5.0(4.0-7.0)\end{array}$ & $\begin{array}{l}n=62 \\
3.0(2.8-5.0)\end{array}$ & $<0.001^{\mathrm{a}}$ \\
\hline $\begin{array}{l}\text { TMT A } \\
\text { Median (IQR) }\end{array}$ & $\begin{array}{l}n=56 \\
49.0(38.0-61.5)\end{array}$ & $\begin{array}{l}n=64 \\
37.5(30.5-52.3)\end{array}$ & $0.008^{\mathrm{a}}$ \\
\hline $\begin{array}{l}\text { TMT B } \\
\text { Median (IQR) }\end{array}$ & $\begin{array}{l}n=43 \\
105.0(84.0-137.0)\end{array}$ & $\begin{array}{l}n=54 \\
72.0(59.0-122.5)\end{array}$ & $0.007^{\mathrm{a}}$ \\
\hline $\begin{array}{l}\text { IS score } \\
\text { Median (IQR) }\end{array}$ & $\begin{array}{l}n=57 \\
84.38(75.00-90.63)\end{array}$ & $\begin{array}{l}n=66 \\
93.75(81.25-100)\end{array}$ & $<0.001^{a}$ \\
\hline
\end{tabular}

${ }^{*} \mathrm{CCl}$ : Charlson Comorbidity Index

${ }^{a}$ Mann-Whitney $U$ test $p$-value

${ }^{\mathrm{b}}$ Fisher's exact test $\mathrm{p}$-value

Note: A sensitivity analyses was carried out with cut-off scores for the API-DM subscale for patient subgroups at 25/75 and at 35/65.The significant variable outcomes across all these analyses are comparable (Additional file 3: supplementary material provided) 


\section{Discussion}

Clinical outcomes associated with RRT modalities are different. The challenge with interpreting reported outcomes on modality superiority are the sociodemographic, physiological and psychological differences which exist between patients in different treatment groups, and the change over time in some of these factors. The systematic exclusion, through lack of information of patient's values, preferences and engagement, leading to the choice (or the lack of one) of modality may also have a bearing on the desired outcomes. In our study of patient preferences for autonomous decisionmaking in ESRD management, we have a large, representative sample population, including predialysis patients in the process of modality decision-making and patients established on 'in-centre' and 'self-care'(predominantly home-based) haemodialysis, from across five tertiary centres. To our knowledge, this is the first study which has examined the issue of patient preferences for information-seeking and decision-making in an ESRD population preparing to receive dialysis and that in receipt of haemodialysis therapy, simultaneously. We have also examined longitudinally, if DM preference in predialysis patients changes over time in a subset of patients, after commencement of dialysis therapy. The variables considered for analyses have been categorised in a manner meaningful for clinical interpretation. These apply especially to neuropsychometric tests and depression and anxiety screening tools where use of cut-off points may result in loss of information to be ascertained from scores further removed from the cut off mark. The coefficient of internal consistency of the two subscales of API is high, ensuring reliability of the test findings in our study population.

In a separate study by Flynn et al.[24], cluster analysis was used to understand the typology of patient preferences in a large group of older adults. The vast majority of them wanted information exchange, but differed in preferences for discussing and selecting treatment choices based on deliberation and decisional control. This study highlighted the need for strategies to improve information exchange and distinguish preferences for discussing and selecting treatment options. Our study has taken the understanding of the subject to individuals who are on a declining health course, those in receipt of different treatment types, respondents in varied sociodemographic groups and considered varied cognitive and psycho-affective factors which may also influence the actual response outcomes.

\section{Demographic variables and patient preference for decision-making in ESRD}

Age is an important factor in decision-making preference, with younger age group preferring a more active role in decision-making. Despite the fact that older patients wanted less participation in treatment decisionmaking, they nonetheless wanted a similar degree of information (high overall median scores on informationseeking subscale), demonstrating that wanting information and making decisions are separate constructs[25]. Even amongst the study cohort of high informationseekers overall, age still emerged as a significant factor for both information-seeking and decision-making, with high scores in favour of lower age. Higher confidence, greater overall perceived knowledge, greater overall retained information/knowledge and access to modern educational resources (internet), consulted by the younger population may play a role, in this regard. Besides, the type of information required by the older age group and the manner in which information is provided to older patients may have to be tailored to individual preferences, such that, their engagement with the decisionmaking process is well facilitated and meaningful. Higher education, like age, was forced into our model for multivariate regression analysis and did emerge as a significant variable for information-seeking, but not for decision-making. This highlights the role of the individual's coping style and the complexity of ESRD decisionmaking process, making collaborative decision-making, a preferred route even amongst those who are well educated and actively seek involvement through information. Even in the cluster examination of 'autonomists' vs 'delegators', education, was not associated with either category. This is contrary to what has been noted in some other conditions where API was used to study decision-making preference [26-28] and also in other qualitative research in the area of medical decisionmaking[29]. Several studies have identified that gender is associated with DM preference, all finding that women are more likely than men to prefer a more active role[30]. This finding has been replicated in our ESRD study population too, although another observational study in ESRD patients, did not find significant gender differences, although a smaller study population may explain this [31]. The role of gender and the biology of decision-making remains an interesting area of research, but, the influence of gender on interpersonal relationship between the physician and the patient may influence participatory decision-making styles [32]. Employment is not a significant predictor on multivariate analysis, but remains a significant association in the cluster of low decision-making scorers. This is likely to be due to the fact that the employment variable is very closely linked to the 'cohort' variable, which was a significant predictor on the regression analysis. The cluster association showed that 'retired' individuals were more likely to assume a passive role in decision-making. Significantly higher proportion (37\%) of unemployed participants were found in the 
'autonomists' group, although the decision to stay unemployed may have been taken consciously by this group. Marital status also seems to influence decision-making preference, with married individuals more likely than unmarried, divorced or widowed participants to play an active role in decision-making. Ethnicity is associated with DM in our study, with white patients more likely to prefer to be involved in decision-making. Patient's role expectations, perceived role in the family context and emphasis on individuality may be a culturally determined phenomenon, influencing the passive role adopted by participants in the ethnic minority group [33]. The approach to imparting information and ascertaining patient's values and preferences should be culturally sensitive and account for the cultural diversity of different regions.

Cognitive function and decision-making preference in ESRD We examined the association of scores from Trail making tests $\mathrm{A}$ and $\mathrm{B}$ and $3 \mathrm{MS}$, a test of global cognition, with decision-making preference scores. The lowest tertile of decision-making scores was associated with poorer scores on the tests of executive brain function. Although this was not significant in the multivariate regression analysis, TMT A scores were significant on single variable analysis. This is likely due to a significant proportion of missing data on TMTs, largely due to patients' inability to complete tests or unwillingness to undertake the tests due to perceived complexity. There is evidence from literature linking age, cognition and other individual resources with health literacy in advanced age[12]. Results show that executive function and episodic memory explained literacy decline with age considerably. Executive function also had an indirect effect via risk aversion. The finding that impaired health literacy in old age is in part a function of cognitive decline even amongst persons without dementia, has clear implications for policy and intervention. Thus, it is high priority to reduce cognitive demands, particularly complex reasoning abilities and memory, inherent in the health literature materials and decision-making aids used by patients with even milder degrees of cognitive impairment. Learning styles specific information and reinforcement of consistent messages will ensure correct understanding. Impact of depression on DM was not significant in our study, but higher BDI scores were significantly associated with higher informationseeking. The ability to appreciate, understand the significance, express choice or engage in a logical process of analysing the information ascertained are known to be impaired in depressed patients in other studies [34].

\section{IIIness burden and decision-making preference in ESRD}

None of the comorbidities emerged significant predictors of decision-making in the multivariate analysis.
However, the Charlson comorbidity index score, a prognostic tool, was significant in the univariate analysis, and so were, diabetes, heart failure and ischaemic heart disease. In the cluster examination, the lowest tertile of decision-making scores was associated significantly with high comorbidity burden. The impact of illness on decision-making is difficult to dissociate from the role of medical care received for the illness on decision-making. Results from studies in published literature suggest that patient's preference may change in time as their experience of illness evolves [35] and that, experiences of interactions with healthcare providers may also affect patient's desire to involve themselves in current or future decision-making[26]. It is apparent from our study that in the small subset of predialysis patients, who were reassessed at least three months after commencement of dialysis therapy, no significant change in their decisionmaking preferences, was observed. It is also apparent that the more complex or urgent the clinical condition, the more likely ESRD patients would consider adopting a more passive role.

\section{Information preference and study group influence}

Our study demonstrates that there is great appetite for information across all study groups. Even amongst the high information-seekers, API information-seeking score greater than 75 , is associated significantly with greater preference for decision-making. The scores are significantly higher in the self-caring cohort and this may well be associated with an active coping style, the same group demonstrating higher preference for involvement in decision-making. The predialysis group was more likely to want shared involvement in the decision-making process compared to other groups in the multivariate analysis and cluster association of low decision-makers. The lack of concrete, personal experience of the treatment process may be the reason for their concern. Therefore, revisiting treatment options after commencement of dialysis may influence the choice of long-term dialysis treatment considerations including location and self vs shared vs institutional care.

\section{Links with medical humanities and social sciences}

The study findings are well in line with predictions drawn from established theories in social psychology. For instance, decision fatigue [36]is a psychological state, where the ability to process complex information and to make autonomous decisions is depleted due to e.g. emotional upheaval, resulting in impulsiveness, evasive behaviour or helplessness. The effects of illness burden on decision-making preference in ESRD can be seen correlating with both decision fatigue and emotional adjustment $[37,38]$ : a patient who has been only recently diagnosed with a severe illness is emotionally and 
cognitively handicapped due to mental fatigue, in comparison to a patient who has had time to process the emotional upheaval and adjust. Recent developments in the psychology of decision-making have revealed several factors influencing and distorting the ability to make autonomous, well-informed decisions, of which decision fatigue is only one. To facilitate patient autonomy in various stages of emotional adjustment and levels of fatigue, procedures including psychological support and appropriate information design become necessary to ensure the fulfillment of patient autonomy.

\section{Practical implications of the knowledge of patient's decision-making preference}

It is known from published literature that patients who are educated about all of their treatment options are significantly more likely to choose a home-based treatment option [39-41]. Information empowers patients to choose their RRT modality. The manner in which this information is presented therefore would influence the patients' choice of therapy. Many decisions of this complexity may well result in a shift in decision-making equipoise, making patient-led autonomous decisions, a function, limited by three key factors-patient characteristics, time constraints and clinical urgency. It is apparent from our study that subsets of well-informed patients are still keen on involving the healthcare team in their decision-making process. There are patient characteristics which influence their wish to be involved in decision-making. These become apparent as the clinical encounter progresses over time. The reasons behind delegating the choice to another person need to be explored at a clinical and psychosociocultural level through collaborative decision-making, engaging patients, their family, and several members of the multi-disciplinary care team. This process typically operates in considerable time constraints, making a truly autonomous decision or shared decision-making by patient choice, an option for a limited few. The third point on clinical urgency is a situation where patients naturally lean towards their physician in making the right choices for them. Presenting all dialysis options as equal with the healthcare team remaining modality neutral (and therefore presumed unbiased), without clarifying the impact of each choice on the course of their illness, associated morbidity, mortality and quality-of-life, renders modality education practice unchanged and unresponsive to published scientific literature. Furthermore, patient's decisionmaking preferences ought to be juxtaposed to the systematic assessment of patients' affect and cognitive abilities and actual as against perceived knowledge. These remain integral to understanding the level and duration of healthcare provider engagement required to facilitate literacy and the decision process.

\section{Study limitations}

There are limitations to our study. Assessing healthcare provider's decision-making preferences would be important as decision-making happens during this bidirectional exchange of information. Assessment of actual knowledge as a predictor of decision-making preference would be useful. Although a number of clinical, psychological and socio-demographic variables have been considered, autonomy preference in a medical context is likely to be influenced by immeasurable factors and therefore our findings do not necessarily present an exhaustive list of predictors of autonomy preference in ESRD or explain the variance in autonomy preference. It is also not possible to ascertain from our study if preferred participation differs from actual participation levels when removed from hypothetical scenarios.

\section{Conclusions}

The study explored decision-making preferences and its influencing factors in ESRD patients overall and according to their position with respect to dialysis commencement. ESRD patients prefer to receive information, but this does not always translate into active involvement in decision-making. This may not be acceptable or appropriate for everyone and the patient may choose to determine the extent to which they seek involvement. By identifying factors which might affect patient preference for involvement, health professionals may move away from a normative, 'one size fits all' approach, be more sensitive to individual patient's preferences and provide better patient-centred; individual-appropriate care.

\section{Additional files}

Additional file 1: SUPPLEMENTARY MATERIAL Missing data

analysis. (DOCX $15 \mathrm{~kb}$ )

Additional file 2: SUPPLEMENTARY MATERIAL-1. TABLE : Item statistics for both subscales. (DOCX $16 \mathrm{~kb}$ )

Additional file 3: Sensitivity Analysis: Cluster Analysis with different API-DM Score cut-offs. (DOCX $29 \mathrm{~kb}$ )

\section{Abbreviations}

3MS: Modified Mini Mental State; API: Autonomy Preference Index; BASIC HHD: Barriers to Successful Implementation of Care in Home Haemodialysis; CCl: Charlson Comorbidity Index; CKD: Chronic Kidney Disease; DM: DecisionMaking; ESRD: End Stage Renal Disease; HD: Haemodialysis; IS: InformationSeeking; NRES: National Research Ethics Service; PD: Peritoneal Dialysis; TMTA/B: Trail Making Tests A/B; BDI: Beck Depression Inventory; STAI: State and Trait Anxiety Inventory.

\section{Competing interests}

The author declare s that he/she has no competing interests.

\section{Authors' contributions}

Research idea and Study design: AJ, SM, PB, AW; Data acquisition- from all centres under supervision of the PIs of the BASIC-HHD study group (Salford Royal NHS Trust - PI: Dr. Grahame Wood, Portsmouth Hospitals NHS Trust - PI: Dr. Juan Mason, University Hospital of North Staffordshire- PI: Dr. Sat Reddy, Ipswich Hospitals NHS Trust- PI: Dr. Praveen Ande); Data analysis/Interpretation: 
AJ, AW, PF, JM, SM. Each author has contributed important intellectual content during manuscript drafting or revision and accepts accountability for the overall work by ensuring all aspects of the work are accurately reported. SM takes responsibility that all study aspects are reported with honesty and transparency. All authors read and approved the final manuscript

\section{Acknowledgements}

We acknowledge the contributions of all research team members who have played crucial role in data acquisition. We would like to thank Ms Jenny Finch for her contribution during data acquisition. We also acknowledge the support of MAHSC, in the conduct of the study.

\section{Funding statement}

The research is funded by Baxter Clinical Evidence Council and NIHR-CLAHRC for Greater Manchester, UK. This funding has been secured through open competition. The study was initiated and designed by the investigators.

\section{Author details}

'Department of Nephrology, Central Manchester Hospitals NHS Trust, Manchester Royal Infirmary, Oxford Road, Manchester M13 9WL, UK. ${ }^{2}$ Department of Political and Economic Studies, University of Helsinki, Helsinki, Finland. ${ }^{3}$ Department of Psychology, University of Manchester, Manchester, UK. ${ }^{4}$ Department of Biostatistics, University of Manchester, Manchester, UK.

Received: 14 March 2015 Accepted: 29 October 2015

Published online: 14 November 2015

\section{References}

1. Tan TZ, Ng GS, Quek C. A Decision Making System Based on Complementary Learning. Intell Syst Ref Libr. 2010;4:163-79.

2. Davis MM, Gorman PN, Rugge JB, Fagan LJ, King VJ, Fagnan LJ. Perception of Shared Decision Making and Clinicians. Med Decis Mak. 2012;32:636-44.

3. Arora NK, Weaver KE, Clayman ML, Oakley-Girvan I, Potosky AL. Physicians' decision-making style and psychosocial outcomes among cancer survivors. Patient Educ Couns. 2009:77(3):404-12.

4. A. Coulter and A. Collins, "MAKING DECISION-MAKING A REALITY No decision about me, without me," Kings Fund, pp. 1-56, 2011.

5. Greenfield S, Kaplan S, Ware Jr JE. Expanding patient involvement in care Effects on patient outcomes. Ann Intern Med. 1985;102(4):520-8.

6. Greenfield S, Kaplan SH, Ware JE, Yano EM, Frank HJ. Patients' participation in medical care: effects on blood sugar control and quality of life in diabetes. J Gen Intern Med. 1988:3(5):448-57.

7. Charles C, Gafni A, Whelan T. Decision-making in the physician-patient encounter: Revisiting the shared treatment decision-making model. Soc Sci Med. 1999;49(5):651-61.

8. Holman $\mathrm{H}$, Lorig K. Patients as partners in managing chronic disease. BMJ $\mathrm{Br}$ Med J. 2000;320(7234):526-7.

9. Guadagnoli E, Ward P. Patient participation in decision-making. Soc Sci Med. 1998:47(3):329-39.

10. Paulus MP. "Neurobiology of decision-making: Quo vadis?", in. Cogn Brain Res. 2005:23(1):2-10

11. Elias MF, Dore GA, Davey A. "Kidney disease and cognitive function,". Contrib Nephrol. 2013:179:42-57.

12. Boyle P a, Yu L, Wilson RS, Segawa E, Buchman AS, Bennett D a. Cognitive decline impairs financial and health literacy among community-based older persons without dementia. Psychol Aging. 2013;28(3):614-24.

13. Baker DW. The meaning and the measure of health literacy. J Gen Intern Med. 2006;21(8):878-83.

14. Murray AM, Tupper DE, Knopman DS, Gilbertson DT, Pederson SL, Li S, et al. Cognitive impairment in hemodialysis patients is common. Neurology. 2006;67(2):216-23.

15. Kimmel PL, Cukor D, Cohen SD, Peterson RA. Depression in end-stage renal disease patients: a critical review. Adv Chronic Kidney Dis. 2007;14(4):328-34.

16. Cukor D, Coplan J, Brown C, Friedman S, Cromwell-Smith A, Peterson RA et al. Depression and anxiety in urban hemodialysis patients. Clin J Am Soc Nephrol CJASN. 2001:2(3):484-90.

17. Ende J, Kazis L, Ash A, Moskowitz MA. Measuring patients' desire for autonomy: decision making and information-seeking preferences among medical patients. J Gen Intern Med. 1989;4(1):23-30.
18. Jayanti A, Wearden AJ, Morris J, Brenchley P, Abma I, Bayer S, et al. "Barriers to successful implementation of care in home haemodialysis (BASIC-HHD):1. Study design, methods and rationale. BMC Nephrol. 2013;14(1):197.

19. Craven JL, Rodin GM, Littlefield C. The Beck Depression Inventory as a screening device for major depression in renal dialysis patients. Int $J$ Psychiatry Med. 1988;18(4):365-74.

20. Spielberger CD. State-Trait Anxiety Inventory. Anxiety. 1987;19:2009.

21. Teng EL, Chui HC. The Modified Mini-Mental State (3MS) examination. J Clin Psychiatry. 1987;48(8):314-8.

22. E. H. Strauss, E. M. S. Sherman, and O. Spreen. A compendium of neuropsychological tests-administration, norms and commentary. Oxford University Press, 3rd Edition, 2006.

23. van Stralen KJ, Dekker FW, Zoccali C, Jager KJ. Confounding. Nephron Clin Pr. 2003;116(2):c143-7.

24. Flynn KE, Smith MA, Vanness D. A typology of preferences for participation in healthcare decision making. Soc Sci Med. 2006;63(5):1158-69.

25. Deber RB. Physicians in health care management: 8 . The patient-physician partnership: decision making, problem solving and the desire to participate. CMAJ. 1994;151(4):423-7.

26. Adams RJ, Smith BJ, Ruffin RE. Patient preferences for autonomy in decision making in asthma management. Thorax. 2001;56(2):126-32.

27. Thompson SC, Pitts JS, Schwankovsky L. Preferences for involvement in medical decision-making: situational and demographic influences. Patient Educ Couns. 1993;22(3):133-40.

28. Nease RF, Brooks WB. Patient desire for information and decision making in health care decisions: the Autonomy Preference Index and the Health Opinion Survey. J Gen Intern Med. 1995;10(11):593-600.

29. Lupton D. Consumerism, reflexivity and the medical encounter. Soc Sci Med. 1997;45(3):373-81.

30. Harwood L, Clark AM. "Dialysis modality decision-making for older adults with chronic kidney disease,". J Clin Nurs. 2014;23(23-24):3378-90.

31. Orsino A, Cameron Jl, Seidl M, Mendelssohn D, Stewart DE. Medical decision-making and information needs in end-stage renal disease patients. Gen Hosp Psychiatry. 2003;25(5):324-31.

32. Kaplan SH, Gandek B, Greenfield S, Rogers W, Ware JE. Patient and visit characteristics related to physicians' participatory decision-making style. Results from the Medical Outcomes Study. Med Care. 1995:33(12):1176-87.

33. Hamann J, Bieber C, Elwyn G, Wartner E, Hörlein E, Kissling W, et al. How do patients from eastern and western Germany compare with regard to their preferences for shared decision making? Eur J Public Health. 2012;22(4):469-73.

34. Hindmarch T, Hotopf M, Owen GS. Depression and decision-making capacity for treatment or research: a systematic review. BMC Med Ethics. 2013;14:54.

35. Butow PN, Maclean M, Dunn SM, Tattersall MHN, Boyer MJ. The dynamics of change: Cancer patients' preferences for information, involvement and support. Ann Oncol. 1997:8(9):857-63.

36. Munro A, Brocas I, Carrillo JD. The psychology of economic decisions, vol. 1: rationality and well-being. J Econ Behav Organ. 2005:58(3):444-7.

37. Lykken DT. Comment on Diener, Lucas, and Scollon (2006). 'Beyond the hedonic treadmill: revising the adaptation theory of well-being'. Am Psychol. 2007;62(6):611-2

38. Diener E, Napa Scollon C, Lucas RE. "The evolving concept of subjective well-being: the multifaceted nature of happiness,". Advances in Cell Aging and Gerontology. 2003;15:187-219.

39. Wuerth DB, Finkelstein SH, Schwetz O, Carey H, Kliger AS, Finkelstein FO. Patients' descriptions of specific factors leading to modality selection of chronic peritoneal dialysis or hemodialysis. Perit Dial Int. 2002;22(2):184-90.

40. Goovaerts T, Jadoul M, Goffin E. Influence of a pre-dialysis education programme (PDEP) on the mode of renal replacement therapy. Nephro Dial Transplant. 2005;20(9):1842-7.

41. Stack AG. Determinants of modality selection among incident US dialysis patients: results from a national study. J Am Soc Nephrol. 2002;13(5):1279-87. 